\title{
Is there a relationship between severity of coronary artery disease and severity of erectile dysfunction?
}

\author{
Lutfi Canat, Gokhan Cicek, Gokhan Atis, Cenk Gurbuz, Turhan Caskurlu \\ Urology Department, Kastamonu State Hospital (LC); Cardiology Department, Kirsehir State Hospital \\ (GC) and Urology Department Istanbul Medeniyet University (GA, CG, TC), Turkey
}

\section{ABSTRACT}

Introduction: The correlation between erectile dysfunction (ED) and coronary artery disease has been emphasized and ED has been recognized as a potential independent risk factor and/or predictor of coronary artery disease (CAD). We evaluated the association between the number of occluded coronary arteries in myocardial infarction (MI) patients with the severity of ED, and investigated the influence of related risk factors in our study group.

Materials and Methods: 183 male patients who underwent coronary angiography because of acute MI from November 2009 to May 2011 were included. Following the stabilization of patients after the treatment, each patient was evaluated for erectile functionality. Risk factors such as age, diabetes, smoking, waist circumference, hypertension, and hematologic parameters were recorded.

Results: Among 183 patients with a mean age of 55.2 years who underwent coronary angiography due to acute MI, 100 (54.64\%) had ED, while the ED rate was 45.36\% (44/97) in cases of single-vessel disease, 64.5\% (31/48) in cases of two-vessel disease, and 65.7\% $(25 / 38)$ in cases of three-vessel disease. The mean IIEF score was $24.2 \pm 4.3,20.4 \pm 4.9$ and $20.5 \pm 4.2$ for single or two or three-vessel disease, respectively. The presence of hypertension aggravated ED only in patients with three-vessel disease and increased total and LDL cholesterol levels in patients with single-vessel or two-vessel disease were accompanied by significantly decreasing IIEF scores.

Conclusion: The severity of ED correlated with the number of occluded vessels documented by coronary angiography, in male patients with acute myocardial infarction. In addition, the presence of hypertension had a significant influence over erectile function only in patients with three-vessel occlusion.

\section{ARTICLE INFO}

\section{Key words:}

Erectile Dysfunction; Coronary Artery Disease;

Coronary Angiography

Int Braz J Urol. 2013; 39: 465-73

Submitted for publication: February 20, 2012

Accepted after revision:

May 25, 2013

\section{INTRODUCTION}

Erectile dysfunction (ED) is the condition of failure to achieve or maintain erection required for sexual intercourse. A recent systemic review found that the age-adjusted incidence of ED was $48.1 \%$ (1). ED is associated with various pathophysiological mechanisms. The 2009 update of the European Association of Urology (EAU) guideline categorizes the causes of ED in 6 main categories: vascular, neurogenic, anatomic/structural, hormonal, psychogenic, and drug-related.

Coronary artery disease (CAD) is the leading cause of death in the Western world, causing serious morbidity rates (2). Diffuse atherosclerotic process affects the arterial blood flow and leads to major pathophysiologic changes involved in both cardiovascular and peripheral arterial diseases including vascular ED (3). Atherosclerosis occurs 
as a result of interaction between environmental and genetic factors. The environmental factors influence the disease progression rate significantly and appear to be the main determinants for the development of both CAD and arterial ED. ED and cardiovascular diseases share some common risk factors such as obesity, cigarette smoking, hypercholesterolemia, metabolic syndrome, and sedentary way of life. Furthermore, among patients demanding a solution for sexual dysfunction, there is a high prevalence of cardiovascular disease, while the potential cardiac risks associated with sexual activity are clearly known. The aim of our study was to investigate the relationship between severity of ED and number of occluded coronary arteries, while evaluating ED in patients with myocardial infarction (MI) who were submitted to angiography.

\section{MATERIALS AND METHODS}

Our study included 183 patients who were submitted to coronary angiography due to acute MI from November 2009 to May 2011. During the angiography, coronary artery disease was defined as an occlusion greater than 50\% in the main coronary artery and/or an occlusion greater than $70 \%$ in any of the other coronary arteries, while the number of occluded arteries was noted. None of the patients included in the study had a history of coronary artery disease or a previous MI attack.

Our study was compliant with the ethical principles laid out by the Helsinki Declaration and written informed consent was obtained from each patient. All patients who stabilized after MI were evaluated by the erectile function domain of the International Index of Erectile Function (IIEF), a validated 15-item self-administered questionnaire, that was translated and validated in Turkish $(4,5)$. The patients were categorized based on their scores as follows: 26-30, no ED; 17-25 mild ED, 11-16 moderate ED, 0-10 severe ED. All included patients in the study were married or had a regular partner.

In order to determine both cardiovascular and ED risk factors, all male patients who underwent angiography were evaluated with regard to parameters such as age, diabetes (defined as a fasting glucose level of $\geq 126$ in two tests), cigarette smoking, waist circumference, hypertension (blood pressure of $\geq 140 / 90 \mathrm{mmHg}$ in 3 consecutive recordings), as well as levels of total cholesterol, LDL, HDL, and triglycerides. The drugs used by the patients (beta blockers, ACE inhibitors, calcium channel blockers, diuretics, nitrates, insulin, oral antidiabetic agents, statins, and acetyl salicyclic acid) were recorded. Patients with other chronic drug use were not included in the study. The patients with a known endocrinologic, neurologic, or psychiatric disease, as well as those who had a history of pelvic-urethral-prostatic surgery, were excluded from the study. Furthermore, those who were under treatment for ED were not included in our study. According to these criteria, a total of 87 patientes were excluded from our study.

The statistical analyses of the study were performed by Kolmogorov-Smirnov test, ANOVA and t test, Kruskal-Wallis test, Mann-Whitney U test, chi-square test, and Fischer's test (Fischer's test was used in cases where chi-square test was not appropriate). Categorical data were studied by Pearson's correlation analysis, whereas discrete data were evaluated by Spearman's correlation analysis.

\section{RESULTS}

Our study included 183 patients who underwent coronary angiography due to acute MI that were categorized in three groups as follows: single-vessel occlusion (Group 1), two-vessel occlusion (Group 2), and three-vessel occlusion (Group 3). Group 1 had 97 patients, whereas Group 2 had 48 patients and Group 3 had 38 patients. The demographic characteristics of the patients are shown in Table- 1 .

The mean age of the studied population was $55.2 \pm 11$ years (range $25-78$ years) and there was no difference between the three groups in terms of age $(p=0.143)$.

Regarding the parameters that might be associated with ED, we found no statistically significant difference between the three groups with regards to DM. The levels of triglycerides, total cholesterol, HDL, and particularly LDL, which is known to be closely related to atherosclerosis, were not significantly different between the studied groups (123.4 mg/dL, $127.9 \mathrm{mg} / \mathrm{dL}, 126.0 \mathrm{mg} /$ 
Table 1 - Demographic characteristics of the patients.

\begin{tabular}{lcccc}
\hline & Group 1 & Group 2 & Group 3 & $p$ \\
\hline Age & $53.14 \pm 11.98$ & $55.04 \pm 11.38$ & $57.42 \pm 10.13$ & 0.143 \\
Waist circumference (cm) & $95.06 \pm 7.00$ & $94.88 \pm 7.27$ & $95.47 \pm 9.42$ & 0.574 \\
Total cholesterol & $194.2 \pm 46.9$ & $194.5 \pm 42.8$ & $198.7 \pm 45.9$ & 0.870 \\
LDL & $123.4 \pm 33.7$ & $127.9 \pm 43.1$ & $126.0 \pm 38.3$ & 0.781 \\
HDL & $39.9 \pm 8.3$ & $38.2 \pm 8.2$ & $40.9 \pm 12.8$ & 0.389 \\
Triglyceride & $166.2 \pm 191.9$ & $152.4 \pm 69.9$ & $161.8 \pm 87.2$ & 0.558 \\
DM (n) & $14.00(14.4 \%)$ & $5.00(10.4 \%)$ & $9.00(23.7 \%)$ & 0.223 \\
HT (n) & $27.00(27.8 \%)$ & $23.00(47.9 \%)$ & $18.00(47.4 \%)$ & 0.021 \\
Cigarette smoking (n) & $70.00(72.2 \%)$ & $33.00(68.8 \%)$ & $30.00(78.9 \%)$ & 0.566 \\
\hline
\end{tabular}

$\mathrm{dL}$, respectively; $\mathrm{p}=0.781$ ). Cigarette smoking, an important risk factor for both cardiovascular disease and ED, was present in most of the patients and had a homogenous distribution betwen the groups $(72.2 \%, 68.8 \%, 78.9 \%$, respectively; $p$ $=0.566)$. Waist circumference was above normal values $(\geq 94 \mathrm{~cm})$ in all groups and there was no significant difference between the groups (Group 1, $95.06 \mathrm{~cm}$; Group 2, $94.88 \mathrm{~cm}$; and Group 3, $95.47 \mathrm{~cm})$. Hypertension (HT), another important risk factor, was significantly higher in two-vessel and three-vessel occlusion groups than in single-vessel occlusion group (Group 1, 27.8\%; Group 2, 47.9\%; Group 3, 47.4\%; $p=0.021$ ).

Use of drugs that could affect the erectile function significantly are shown in detail in Table-2. Of 183 patients diagnosed with acute MI, 100 (54.64\%) had varying degrees of ED (IIEF score $<26$ ), while ED relative rates of the groups was 45.36\% (44/97) for Group 1, 64.5\% (31/48) for Group 2, and 65.7\% (25/38) for Group 3. ED rate in single-vessel occlusion group was signifi- cantly lower than in two-vessel and three-vessel occlusion groups ( $\mathrm{p}<0.001)$. The mean IIEF score was $24.2 \pm 4.3$ in Group 1, $20.4 \pm 4.9$ in Group 2 , and $20.5 \pm 4.2$ in Group 3. Although the mean IIEF score was significantly lower in two-vessel and three-vessel occlusion groups than in single-vessel occlusion group ( $p=0.001)$, there was no significant difference between the two-vessel and three-vessel occlusion groups in relation to IIEF scores (Table-3).

The relation of aging with severity of CAD and erectile functions was studied by multivariate analysis. Intragroup analyses revealed a significant decrease in IIEF scores with increasing age in all three groups, while increasing age was found to affect erectile functions similarly in all groups (Table-4).

Evaluation of the relationship between $\mathrm{ED}$, severity of CAD, and DM demonstrated that there was no significant difference between the IIEF scores of patients with and without DM and the number of occluded vessels (Table-5). 
Table 2 - Situations of chronic drug use before MI.

\begin{tabular}{lcccc}
\hline & Group 1 & Group 2 & Group 3 & p \\
\hline Beta blocker & $13.00(13.4 \%)$ & $8.00(16.7 \%)$ & $10.00(26.3 \%)$ & 0.198 \\
Diuretic & $1.00(1.0 \%)$ & $1.00(2.1 \%)$ & $10.00(26.3 \%)$ & 0.651 \\
ACE inhibitor & $16.00(16.5 \%)$ & $10.00(20.8 \%)$ & $14.00(36.8 \%)$ & 0.036 \\
OAD & $8.00(8.2 \%)$ & $2.00(4.2 \%)$ & $8.00(21.1 \%)$ & 0.025 \\
Insulin & $1.00(1.0 \%)$ & $2.00(4.2 \%)$ & $3.00(7.9 \%)$ & 0.121 \\
Nitrate & $1.00(1.0 \%)$ & $3.00(6.3 \%)$ & $4.00(10.5 \%)$ & 0.040 \\
Statin & $4.00(4.1 \%)$ & $5.00(10.4 \%)$ & $5.00(13.2 \%)$ & 0.145 \\
ASA & $16.00(16.5 \%)$ & $7.00(14.6 \%)$ & $7.00(18.4 \%)$ & 0.867 \\
Calcium channel blocker & $5.00(5.2 \%)$ & $2.00(4.2 \%)$ & $4.00(10.5 \%)$ & 0.409 \\
\hline
\end{tabular}

ACE: Angiotensin converting enzyme; OAD: Oral antidiabetics; ASA: Acetylsalicylic Acid

Table 3 - IIEF scores relative to the groups.

\begin{tabular}{lcccc}
\hline & Group 1 & Group 2 & Group 3 & $p$ \\
\hline IIEF score & $24.2 \pm 4.3$ & $20.4 \pm 4.9$ & $20.5 \pm 4.2$ & 0.001 \\
\hline
\end{tabular}

Table 4 - IIEF scores relative to age groups and number of occluded vessels.

\begin{tabular}{lccc}
\hline & Group 1 & Group 2 & Group 3 \\
\hline$<40$ years & $27.73 \pm 0.65$ & $24.00 \pm 2.24$ & $24.50 \pm 0.71$ \\
$40-50$ years & $25.50 \pm 3.53$ & $23.82 \pm 0.87$ & $21.00 \pm 5.66$ \\
$50-60$ years & $24.34 \pm 2.87$ & $19.35 \pm 5.02$ & $20.92 \pm 3.96$ \\
$60-70$ years & $20.50 \pm 3.42$ & $19.89 \pm 3.76$ & $18.85 \pm 3.72$ \\
$>70$ years & $18.89 \pm 6.49$ & $15.17 \pm 6.24$ & $18.80 \pm 2.70$ \\
\hline
\end{tabular}


Table 5 - The relationship between IIEF scores and number of occluded vessels in terms of diabetic status.

\begin{tabular}{lccc}
\hline & DM $(-)$ & DM $(+)$ & $P$ \\
\hline Group 1 & $24.45 \pm 4.14$ & $22.57 \pm 4.88$ & 0.059 \\
Group 2 & $20.56 \pm 4.88$ & $19.40 \pm 5.32$ & 0.620 \\
Group 3 & $20.90 \pm 3.99$ & $19.22 \pm 4.79$ & 0.301 \\
\hline
\end{tabular}

Table 6 - The relationship between IIEF scores and number of occluded vessels in terms of hypertension.

\begin{tabular}{lccc}
\hline & HT $(-)$ & HT $(+)$ & $p$ \\
\hline Group 1 & $24.49 \pm 4.21$ & $23.37 \pm 4.41$ & 0.091 \\
Group 2 & $21.08 \pm 4.82$ & $19.74 \pm 4.95$ & 0.347 \\
Group 3 & $21.85 \pm 3.39$ & $19.00 \pm 4.56$ & 0.034 \\
\hline
\end{tabular}

Evaluation of the relationship between severity of $\mathrm{CAD}$, erectile function, and $\mathrm{HT}$ revealed that erectile function was affected negatively in patients with three-vessel occlusion and HT ( $\mathrm{p}=$ 0.034), whereas there was no significant correlation between HT and IIEF scores in single-vessel and two-vessel groups (Table-6).

Evaluation of the influence of cigarette smoking over erectile function among our studied groups revealed that cigarette smoking had a similar impact on erectile function in all groups (Table-7).
There was no significant relationship between waist circumference and erectile function in our studied groups. Multivariate analyses based on the entire laboratory data demonstrated a significant correlation only between total cholesterol and LDL cholesterol levels in Group 1 and Group 2, whereas increasing total and LDL cholesterol levels in patients with single-vessel or two-vessel occlusion were accompanied by significantly decreasing IIEF scores, however, no such significant correlation was observed in Group 3. The multivariate analysis investigating the relationship of erectile function

Table 7 - The impact of cigarette smoking and number of occluded vessels on IIEF scores.

\begin{tabular}{lccc}
\hline & Cigarette smoking $(-)$ & Cigarette smoking $(+)$ & $p$ \\
\hline Group 1 & $21.96 \pm 5.41$ & $24.41 \pm 3.43$ & 0.101 \\
Group 2 & $19.67 \pm 5.26$ & $20.79 \pm 4.74$ & 0.324 \\
Group 3 & $19.38 \pm 4.53$ & $20.80 \pm 4.12$ & 0.400 \\
\hline
\end{tabular}


with current medication and number of occluded vessels revealed no significant correlation.

\section{DISCUSSION}

The most frequently mentioned risk factors of ED are also defined as risk factors of atherosclerosis and eventually CAD. In addition to sharing common risk factors, these two conditions demonstrate similar characteristics such as impaired endothelial function and higher prevalence of ED in men with vascular disease. While endothelial dysfunction has been shown to be present in many ED patients, various events associated with atherosclerosis or artery-related pathology have been shown to be related to ED as well. For instance, many organic EDs have been demonstrated to arise from arteriogenic factors; among organic factors, $40.5 \%$ have been shown to be of arterial origin, $10 \%$ of cavernosal origin, whereas 12.5\% and $1.8 \%$ have been shown to be associated with neurogenic ED and endocrinologic ED, respectively (6). Recently, the most commonly investigated subject has been the possibility of ED being a predictor or risk factor of cardiovascular diseases. COBRA trial demonstrated that ED arises before $\mathrm{CAD}$ in the majority by an average of 2 up to 3 years (7). Moreover, one of the recommendations of the The Princeton III consensus was all men with ED who are older than 30 years with increased CVD risk should be submitted to a thorough noninvasive and, when indicated, invasive evaluation of CVD status (8).

Epidemiologically, adjusted to world standard population, prevalence rate of ED in the United States of America was 33.7\% in the year 2011, in contrast to the adjusted prevalence in the Middle East (47\%) (9). In another prevalence study, overall prevalence of ED was found to be $69.2 \%$ (33.2\%, mild; 27.5\% moderate; 8.5\%, severe ED) and it was observed to rise with increasing age (10). In our study, IIEF scores demonstrated a decline with increasing age in acute MI patients with single-, two-, or three-vessel disease.

Wabrek and Burchell studied 131 men (age: 31-86 years) admitted for acute MI and found that $64 \%$ of them were impotent (11). Sjogren et al. compared pre- and post-MI ED prevalences in 49 men and determined that ED prevalence increased to $45 \%$ from $18 \%$, while the new onset or progressing ED prevalence in this group of men was 43\% (12). These two studies constitute the basis of the following studies on CAD-ED relationship.

In this study, 54.64\% of 183 patients diagnosed with acute MI had varying degrees of ED, whereas this rate was $45.36 \%$ in the single-vessel group, 64.5\% in the two-vessel group, and 65.7\% in the three-vessel group. Salem et al. conducted a case-control study on prevalence rates comparing newly diagnosed 183 coronary artery patients and 134 controls wherein ED prevalence was $88.5 \%$ in cases with CAD and $64.2 \%$ in cases without CAD ( $p<0.05$ ). They concluded that presence of ED might be an alternative marker for CAD and that severe ED was an independent risk factor of CAD (13). However, in their study, the relationship between CAD and severity of coronary artery disease was not mentioned. In consistance with our study, El-Sakka et al. reported a significant correlation between ischemic heart disease and erectile dysfunction. In their study, among 417 patients with ED, 27.6\% had mild, 30\% had moderate, and $42.4 \%$ had severe ED, $26.9 \%$ of the patients were found to have varying degrees of ischemic heart disease and severity of ED was observed to be significantly elevated in patients with ischemic heart disease of high degree (14). However, Montorsi et al. detected ED in $49 \%$ of 300 coronary artery patients with acute chest pain submitted to angiography, while no significant difference was found between patients with and without ED with regard to angiographic characteristics (15).

Chew et al. conducted a retrospective study based on the hospital data wherein they investigated the relationship between ED and subsequent cardiovascular events in 1660 cases. They found that the incidence of atherosclerotic cardiovascular disease was statistically significantly higher in ED patients. There was a correlation between ED and atherosclerotic cardiovascular disease in all the groups of patients under 70 years of age. They concluded that ED was a strong predictor of atherosclerotic cardiovascular disease and that early age in ED patients was an even stronger predictor of cardiovascular event (16). Another prospective study, which also clearly revealed the relationship 
between ED and cardiovascular disease, investigated the correlation between ED and impaired myocardial perfusion based on myocardial perfusion scintigraphy images of 287 patients. As $47.8 \%$ of the patients had varying degrees of ED, the incidence of impaired myocardial perfusion and reduced left ventricular wall motion was observed to be significantly higher in patients with ED (17).

In our study, unlike many other studies, the relationship between number of involved coronary arteries and severity of ED was investigated with regard to some demographic characteristics and atherosclerotic risk factors. First, we studied the relationship between number of involved coronary arteries and erectile function at various ages. Our study revealed a significant decrease in IIEF scores with increasing age, independent of the severity of coronary artery disease, whereas increasing age was observed to have an equal impact on erectile function in single-, two-, and three-vessel occlusion groups.

ED is a common complication of type 2 $\mathrm{DM}$, and certain risk factors are associated with the presence of ED and severity (18). Age-adjusted incidence of complete ED has been found to be 3-fold higher in men with DM than in men without DM (19). We performed a literature review on studies investigating ED-CAD-DM relationship in consideration of the fact that DM is also a serious risk factor of CAD. Gaccaruso et al. studied 293 diabetic patients and revealed that after adding erectile dysfunction to the list of risk factors for CAD mentioned in recent guidelines, the sensitivity of these risk factors for asymptomatic CAD in diabetic patients rised to $89 \%$ from $62 \%$. This study has shown that ED is a strong predictor of CAD in diabetic patients (20). Another study comprised of a small group of type 2 diabetes patients showed that total cardiovascular risk was significantly higher in patients with ED than in those without ED ( $p=0.002)$. Similarly, 10-year coronary and cardiac risk as well as vascular and atherosclerotic risk were found to be significantly higher in patients with ED as well. The study concluded that increasing severity of erectile dysfunction caused a significant increase in total cardiovascular risk in diabetic patients (21). In another comprehensive study focusing on the same subject, the relationship between erectile problem and cardiovascular disease-related mortality in type 2 diabetes patients was investigated in 6304 male individuals. Initially, ED patients were found to be under higher risk of cardiovascular disease (hazard ratio 1.19, 95\% CI 1.08 to 1.32). Similarly, patients with ED demonstrated an increased risk of coronary heart disease as well (hazard ratio 1.35, 95\% CI 1.16 to 1.56). Furthermore, increasing risk was observed to reach the peak point 2 years after the onset of ED (22). Unlike other trials, our study focused on the relationship between erectile function and severity of coronary artery disease in diabetic patients. In conclusion, we observed no significant difference between number of involved vessels and erectile function of diabetic or non-diabetic individuals.

Another factor involved in the etiology of ED is hypertension. The relationship between HT and CAD have been investigated by many researchers and coronary artery disease diagnosed with angiography has been shown to have a strong correlation with systolic blood pressure and pulse pressure (23). A study in Egypt found hypertension, unrelated to their ongoing treatment, in 17.3\% of 658 patients with ED. Multivariate analysis demonstrated that HT increased ED risk by 5.4-fold (24). In our study, as the number of occluded coronary arteries increased, incidence of HT was observed to rise, as well. According to the multivariate analysis of ED relative to these two variables, HT presence was observed to have a more negative effect on erectile function in patients with three-vessel occlusion. In patients with single- and two-vessel occlusion, no significant correlation was found between HT and erectile function. In conclusion, CAD may be more severe in ED patients with HT.

Tobacco use has been definitely shown to cause damage particularly in the arterial endothelium with microvascular structure. Therefore, cigarette smoking can be regarded as a risk factor that can lead to sexual dysfunction. Today, although the inadequacy of researches revealing the direct relationship between cigarette smoking and ED is mentioned, many recent studies show that cigarette smoking significantly increases the risk of ED. Wu et al. conducted a prevalence study 
on a large case series comprising 2686 patients and the prevalence of ED was found to be $49.5 \%$ between 20-79 years of age. Furthermore, ED prevalence was observed to be significantly higher in patients that smoke more than 20 cigarettes a day than in non-smokers (OR 1.23, 95\% CI 1.03-1.49, $\mathrm{P}=0.02$ ). However, the highest risk of ED in cigarette smokers, as compared with the non-smokers, was observed after 23 years (25). In another study, $40.1 \%$ of 658 ED patients and $28.7 \%$ of 821 controls were found to be smokers, whereas smoking was determined to elevate the risk of ED by 3.1fold (24). However, none of those studies mentioned a relationship of ED with smoking cigarette and CAD severity. In our study, we determined no significant relationship between cigarette smokers and non-smokers with regard to erectile function in the single-, two-, and three-vessel occlusion groups. However, we believe that this result should be verified by studies including larger series.

Obesity, another common risk factor of both ED and CAD, and particularly abdominal visceral fat deposition, has been shown by many studies to increase cardiovascular disease and early mortality risk. Furthermore, its relationship with ED has been clearly demonstrated, as well. It is a well known fact that waist circumference is directly related to ED and that central obesity increases the risk of cardiovascular disease, therefore, measuring waist circumference is regarded as an important potential predictor of ED (26). Another effect of central obesity over erectile function is the reduction of testosterone levels caused by some endocrinologic changes (increasing insulin, glucose, and C peptide concentrations). Corona et al. conducted a study to reveal the relationship between obesity, testosterone, and major cardiovascular disease in a series of 1687 cases and showed that obesity and obesity-related hypogonadism were associated with increased incidence of major cardiovascular events (27). In our study, absence of post-MI testosterone measurements can be regarded as a limitation of our study. However, we were able to show that erectile function was not correlated with the number of occluded vessels and waist circumference.

According to the lipid profiles of the patients, increased total and LDL cholesterol levels were observed to be correlated to significant decrease of IIEF scores in single- and two-vessel occlusion groups. In other words, ED risk was found to rise with increasing cholesterol levels in patients with coronary artery disease. However, this correlation was not present in patients with three-vessel occlusion. Although Chang et al. found a strong correlation between ED and low HDL cholesterol level in ED cases (28), we did not find such a relationship in our study.

Sample size of our study was relatively small. Since our study did not include many patients in the multivariate subgroups, we believe that similar studies based on larger series, including long-term follow-up results of erectile function, should be performed.

\section{CONCLUSIONS}

In the present study, erectile function after acute MI was significantly poorer in patients with two- and three-vessel occlusion than in patients with single-vessel occlusion. In addition, the presence of hypertension was observed to have a further negative effect on erectile function in patients with three-vessel occlusion. Moreover, increasing total and LDL cholesterol levels were found to be associated with significant deterioration in erectile function in patients with single- and two-vessel occlusion.

\section{CONFLICT OF INTEREST}

None declared.

\section{REFERENCES}

1. Teles AG, Carreira M, Alarcão V, Sociol D, Aragüés JM, Lopes $\mathrm{L}$, et al.: Prevalence, severity, and risk factors for erectile dysfunction in a representative sample of 3,548 portuguese men aged 40to 69 years attending primary healthcare centers: results of the Portuguese erectile dysfunction study. J Sex Med. 2008; 5: 1317-24.

2. Harriss LR, Ajani AE, Hunt D, Shaw J, Chambers B, Dewey $\mathrm{H}$, et al:: Accuracy of national mortality codes in identifying adjudicated cardiovascular deaths. Aust N Z J Public Health. 2011; 35: 466-76.

3. Krane RJ, Goldstein I, Saenz de Tejada I: Impotence. N Engl J Med. 1989; 321: 1648-59. 
4. Cappelleri JC, Rosen RC, Smith MD, Mishra A, Osterloh IH: Diagnostic evaluation of the erectile function domain of the International Index of Erectile Function. Urology. 1999; 54: 346-51.

5. Turunç T, Deveci S, Güvel S, Peşkircioğlu L: The assessment of Turkish validation with 5 question version of internation index of erectile function. Turkish J Urol. 2007; 33: 45-9.

6. Caskurlu T, Tasci Al, Resim S, Sahinkanat T, Ergenekon E: The etiology of erectile dysfunction and contributing factors in different age groups in Turkey. Int J Urol. 2004; 11: 525-9.

7. Montorsi P, Ravagnani PM, Galli S, Rotatori F, Veglia F, Briganti $A$, et al.: Association between erectile dysfunction and coronary artery disease. Role of coronary clinical presentation and extent ofcoronary vessels involvement: the COBRA trial. Eur Heart J. 2006; 27: 2632-9.

8. Nehra A, Jackson G, Miner M, Billups KL, Burnett AL, Buvat J, et.: The Princeton III Consensus recommendations for the management of erectile dysfunction and cardiovascular disease. Mayo Clin Proc. 2012; 87: 766-78.

9. Shaeer 0, Shaeer K: The Global Online Sexuality Survey (GOSS): the United States of America in 2011. Chapter I: erectile dysfunction amongEnglish-speakers. J Sex Med. 2012; 9: 3018-27.

10. Akkus E, Kadioglu A, Esen A, Doran S, Ergen A, Anafarta K, et al.: Prevalence and correlates of erectile dysfunction in Turkey: a population-based study. Turkish Erectile Dysfunction Prevalence Study Group. Eur Urol. 2002; 41: 298-304.

11. Wabrek AJ, Burchell RC: Male sexual dysfunction associated with coronary heart disease. Arch Sex Behav. 1980; 9: 69-75.

12. Althof SE: Quality of life and erectile dysfunction. Urology. 2002; 59: 803-10.

13. Salem S, Abdi S, Mehrsai A, Saboury B, Saraji A, Shokohideh $V$, et al.: Erectile dysfunction severity as a risk predictor for coronary artery disease. J Sex Med. 2009; 6: 3425-32.

14. El-Sakka AI, Morsy AM, Fagih BI, Nassar AH: Coronary artery risk factors in patients with erectile dysfunction. J Urol. 2004l; 172: 251-4.

15. Montorsi F, Briganti A, Salonia A, Rigatti P, Margonato A, Macchi $A$, et al.: Erectile dysfunction prevalence, time of onset and association with risk factors in 300 consecutive patients with acute chestpain and angiographically documented coronary artery disease. Eur Urol. 2003; 44: 360-4; discussion 364-5.

16. Chew KK, Finn J, Stuckey B, Gibson N, Sanfilippo F, Bremner A, et al.: Erectile dysfunction as a predictor for subsequent atherosclerotic cardiovascular events: findings from a linkeddata study. J Sex Med. 2010; 7: 192-202.

17. Ortiz J, Ortiz ST, Monaco CG, Yamashita CH, Moreira MC, Monaco CA: Erectile dysfunction: a marker for myocardial perfusion impairment?. Arq Bras Cardiol. 2005; 85: 241-6.

18. Yang G, Pan C, Lu J: Prevalence of erectile dysfunction among Chinese men with type 2 diabetes mellitus. Int $\mathrm{J}$ Impot Res. 2010; 22: 310-7.
19. Kupelian V, Shabsigh R, Araujo AB, O'Donnell AB, McKinlay $\mathrm{JB}$ : Erectile dysfunction as a predictor of the metabolic syndrome in aging men: results from the Massachusetts Male AgingStudy. J Urol. 2006; 176: 222-6.

20. Gazzaruso C, Coppola A, Montalcini T, Valenti C, Garzaniti A, Pelissero G, et al.: Erectile dysfunction can improve the effectiveness of the current guidelines for the screening for asymptomatic coronary artery disease in diabetes. Endocrine. 2011; 40: 273-9.

21. Meena BL, Kochar DK, Agarwal TD, Choudhary R, Kochar A: Association between erectile dysfunction and cardiovascular risk in individuals with type-2 diabetes without overtcardiovascular disease. Int J Diabetes Dev Ctries. 2009; 29: 150-4.

22. Batty GD, Li Q, Czernichow S, Neal B, Zoungas S, Huxley R, et al.: Erectile dysfunction and later cardiovascular disease in men with type 2 diabetes: prospective cohort study based on theADVANCE (Action in Diabetes and Vascular Disease: Preterax and Diamicron Modified-Release Controlled Evaluation) trial. J Am Coll Cardiol. 2010; 56: 1908-13.

23. Mayer B, Lieb W, Radke PW, Götz A, Fischer M, Bässler A, et al.: Association between arterial pressure and coronary artery calcification. J Hypertens. 2007; 25: 1731-8.

24. Zedan H, Hareadei AA, Abd-Elsayed AA, Abdel-Maguid EM: Cigarette smoking, hypertension and diabetes mellitus as risk factors for erectile dysfunction in upper Egypt. East Mediterr Health J. 2010; 16: 281-5.

25. Wu C, Zhang H, Gao Y, Tan A, Yang X, Lu Z, et al.: The association of smoking and erectile dysfunction: results from the Fangchenggang Area Male Health and ExaminationSurvey (FAMHES). J Androl. 2012; 33: 59-65.

26. Demir T, Demir 0, Kefi A, Comlekci A, Yesil S, Esen A: Prevalence of erectile dysfunction in patients with metabolic syndrome. Int J Urol. 2006; 13: 385-8. Erratum in: Int J Urol. 2006; 13: 1264. Demir, Omer [added]; Kefi, Aykut [added]; Comlekci, Abdurrahman [added]; Yesil, Sena [added]; Esen, Adil [added].

27. Corona G, Rastrelli G, Monami M, Melani C, Balzi D, Sforza A, et al.: Body mass index regulates hypogonadism-associated CV risk: results from a cohort of subjects with erectile dysfunction. J Sex Med. 2011; 8: 2098-105.

28. Chang ST, Chu CM, Hsu JT, Hsiao JF, Chung CM, Ho C, et al.: Independent determinants of coronary artery disease in erectile dysfunction patients. J Sex Med. 2010; 7: 1478-87. 